\title{
Acute postoperative opioid consumption trajectories and long-term outcomes in pediatric patients after spine surgery
}

This article was published in the following Dove Press journal:

Journal of Pain Research

\author{
Mandy MJ Li, ${ }^{1,2}$ \\ Don Daniel Ocay ${ }^{2,3}$ \\ Alisson R Teles ${ }^{2,4}$ \\ Pablo $M$ Ingelmo $0^{5,6}$ \\ Jean A Ouellet ${ }^{1,2,7}$ \\ M Gabrielle Pagé 8,9 \\ Catherine E Ferland ${ }^{2,4-6,10}$ \\ 'Faculty of Medicine, McGill University, \\ Montreal, Quebec, Canada; ${ }^{2}$ Department \\ of Clinical Research, Shriners Hospitals \\ for Children-Canada, Montreal, Quebec, \\ Canada; ${ }^{3}$ Department of Experimental \\ Surgery, McGill University, Montreal, \\ Quebec, Canada; ${ }^{4}$ Integrated Program in \\ Neurosciences, McGill University, \\ Montreal, Quebec, Canada; ${ }^{5}$ Chronic \\ Pain Services, Montreal Children's \\ Hospital, Montreal, Quebec, Canada; \\ ${ }^{6}$ Department of Anesthesia, McGill \\ University, Montreal, Quebec, Canada; \\ ${ }^{7}$ Division of Orthopaedic Surgery, McGill \\ University, Montreal, Quebec, Canada; \\ ${ }^{8}$ Département d'anesthésiologie, \\ Université de Montréal, Montreal, \\ Quebec, Canada; ${ }^{9}$ Carrefour de l'innova- \\ tion et de l'évaluation en santé, Centre \\ de recherche du Centre hospitalier de \\ I'Université de Montréal (CRCHUM), \\ Montreal, Quebec, Canada; ${ }^{10}$ Child \\ Health and Human Development \\ Research Axis, Research Institute-McGill \\ University Health Centre, Montreal, \\ Quebec, Canada
}

Correspondence: Catherine E Ferland Shriners Hospitals for Children-Canada, I003, Boul. Décarie, Montréal H4A 0A9, Canada

Tel + I 5I4842 4464 ext $7 \mid 77$

Fax + I 514842 558I

Email catherine.ferland@mcgill.ca
Background: The days following surgery are a critical period where the use of opioids predicts long-term outcomes in adults. It is currently unknown as to whether opioid consumption throughout the acute postoperative period is associated with long-term outcomes in pediatric patients. The aims of this study were to characterize opioid consumption trajectories in the acute postoperative period, identify predictors of trajectory membership and determine associations between opioid consumption trajectories and long-term patient outcomes.

Materials and methods: Medication use, pain and mental health status were assessed at baseline in adolescents with idiopathic scoliosis who were scheduled for spinal fusion surgery. Cumulative 6-hr opioid consumption was recorded for up to 5 days after spinal surgery. At 6 months after surgery, medication use, pain and functional activity were evaluated. Growth mixture modeling was used to identify opioid trajectories.

Results: One hundred and six patients were included in the study. Mean cumulative 6-hr opioid consumption in the acute postoperative period was $13.23 \pm 5.20 \mathrm{mg} / \mathrm{kg}$. The model with the best fit contained 5 acute postoperative trajectories and a quadratic term (AIC $=6703.26$, BIC $=6767.19$ ). Two types of patient behaviors were identified: high opioid consumers (trajectories 4 and 5) and low opioid consumers (trajectories 1, 2 and 3). Intraoperative intrathecal morphine dose was a predictor of trajectory membership $(p=0.0498)$. Opioid consumption during the acute postoperative period was not significantly associated with pain, functional activity or pain medication use at 6 months after surgery.

Conclusion: In pediatric patients, intraoperative intrathecal morphine dose predicts opioid consumption in the acute postoperative period. Importantly, opioid consumption during this period does not affect long-term outcomes in pediatric patients after a spine surgery.

Keywords: opioids, pediatrics, postsurgical pain, trajectories, adolescent idiopathic scoliosis, spinal fusion surgery

\section{Introduction}

Opioids are the mainstay method of pain management in the acute postoperative period. ${ }^{1}$ The problem with this practice is 2-fold: (1) opioid-induced adverse events such as respiratory depression are serious and life-threatening, ${ }^{2}$ and (2) acute opioid exposure after surgery increases the risk of chronic opioid use. ${ }^{3}$ With over 300,000 children and adolescents undergoing surgery in Canada and the United states each year, postoperative opioid exposure is a prevalent issue. ${ }^{4,5}$

The days following surgery are a critical period where the progression of opioid consumption has the potential to influence long-term outcomes. Most studies 
examining the acute postoperative period in the pediatric population focused on total opioid consumption over the in-hospital period. ${ }^{6-8}$ However, these studies omitted to report consideration of the evolution of opioid intake over time. Recently, Kwan et $\mathrm{al}^{9}$ reported opioid intake at $12-24 \mathrm{hr}$ intervals for up to 2 weeks after surgery in a cohort of adolescent idiopathic scoliosis (AIS) patients. While opioid consumption was considered in relation to time, variability existed in the mean analgesic intake at each timepoint, suggesting heterogeneity of the study population and the presence of multiple subgroups.

Recently trajectory analysis has emerged as a method to capture longitudinal change of a variable over time. Given that populations are not homogenous, latent subgroups with unique opioid consumption trajectories may be elucidated using this method. Highlighting the practical application of trajectory analysis, Pagé et $\mathrm{al}^{10}$ identified 4 pain trajectories in the acute postoperative period in adults after total hip arthroplasty. Importantly, preoperative variables such as pain and anxiety predicted pain trajectory membership. Pain trajectories were in turn associated with long-term outcomes such as pain and functional disability. ${ }^{10}$ Recently, Pagé et al ${ }^{11}$ identified 4 pain trajectories and 4 in-hospital opioid consumption trajectories among patients undergoing hepatic resection. Trajectory membership in this case did not predict chronic postsurgical pain at 6 months but predicted patients' levels of pain catastrophizing at follow-up.

To the best of our knowledge, no studies have been done to examine opioid consumption trajectories in the acute postoperative period in the pediatric patient population. Therefore, the aims of this study were to (1) identify opioid consumption trajectories in the acute postoperative period, (2) identify predictors of opioid consumption trajectories and (3) identify associations between trajectory membership and long-term patient outcomes in a cohort of AIS patients undergoing spinal fusion surgery.

\section{Materials and methods}

This study was part of a larger study assessing perioperative pain in children undergoing orthopedic surgery. Ethics approval was obtained prior to the beginning of the study from the Research Ethics Board of McGill University (A08M71-17B).

\section{Study participants}

Patients between the ages of 10 and 18 years with AIS were recruited from the outpatient spine clinic of the Shriners Hospitals for Children-Canada between 2013 and 2018. Prior to the beginning of the study, written informed patients' consents were obtained. In patients under the age of 18 , written informed consent was obtained from a parent or legal guardian. The study was conducted in accordance with the Declaration of Helsinki. Inclusion criteria included adolescents diagnosed with AIS and scheduled to undergo first time posterior spinal fusion (PSF) surgery with instrumentation. Exclusion criteria included children with neuromuscular scoliosis, children unable to speak, write or read English or French, children diagnosed with intellectual disability that would interfere with understanding of questions being asked and children with major chronic medical conditions (American Society of Anesthesiology status III or higher). Research assistants collected information on demographics, medical history and selfreported questionnaires. A total of 126 patients consented to participate in the study. Prior to surgery, 8 patients were excluded from analysis due to drop out $(\mathrm{n}=6)$ and incomplete baseline questionnaires $(\mathrm{n}=2)$. After surgery, an additional 12 patients were excluded due to drop out $(\mathrm{n}=1)$, surgical complications $(\mathrm{n}=5)$, deviation from the anesthesia protocol $(\mathrm{n}=2)$, incomplete postoperative data $(\mathrm{n}=2)$ and lost to follow-up $(n=2)$. The final opioid consumption trajectory analysis included 106 study participants. Out of the 106, only 76 patients were analyzed for long-term patient outcomes at 6 months due to loss to follow-up ( $\mathrm{n}=30$ ). Patients dropped out of the study or were lost to follow-up for one of the following reasons: patients were no longer interested in participating in the study, patients were unable to attend the 6-month follow-up appointment due to distance/difficulty of travel or patients had not yet attended their scheduled follow-up appointment at the time of the data analysis.

\section{Experimental design}

Study variables were assessed at different timepoints throughout the perioperative period: baseline (7-10 days before surgery during the standard preoperative consultation at the hospital); postoperative hours (POH) 1 to 120 from the moment the patient left the operating room; and follow-up (6 months after surgery).

\section{Questionnaires}

At baseline and at the 6-month follow-up appointment, patients were asked to complete the Scoliosis Research Society-30 (SRS-30) questionnaire. The SRS-30 has been validated for patients with AIS and is one of the most widely used tools in assessing the quality of life and spinal surgery outcomes for individuals with scoliosis. ${ }^{12,13}$ At the same timepoints, patients were also asked to indicate their medication use for pain from 
the following options: none, non-opioids (eg, Tylenol, ibuprofen) or opioids (eg, Dilaudid).

\section{Perioperative anesthesia care}

Perioperative analgesia was standardized including an intraoperative anesthesia institutional standardized protocol specific to the study. All patients received total intravenous anesthesia with propofol, remifentanil/sufentanil/fentanyl, ketamine and dexamethasone. As per our standard of care, after induction, all patients received an intrathecal injection of morphine, with a suggested dose of $5 \mu \mathrm{g} / \mathrm{kg}$, although at the discretion of the anesthesiologists. After surgery, all patients received IV patient-controlled analgesia (PCA) morphine/ketamine (1:1) $20 \mu \mathrm{g} / \mathrm{kg}$ bolus on demand, 6-min lockout interval, maximum dose per hour $0.1-0.4 \mathrm{mg} / \mathrm{kg} / \mathrm{hr}$ available upon arrival at the post-anesthesia care unit (PACU). The Acute Pain Services (APS) team independently evaluated the patients twice daily and adjusted the dosage based on the patient response and adverse effects. If a patient presented significant postoperative nausea and vomiting on postoperative day 2 or 3 , the APS team would change the analgesic solution to hydromorphone/ketamine (bolus $4 \mu \mathrm{g} / \mathrm{kg}$, lock out 6 mins, maximum dose per hour $0.02-0.08 \mathrm{mg} / \mathrm{kg}$ ). In addition, all patients received acetaminophen $15 \mathrm{mg} / \mathrm{kg}$ Q6 hrs, IV ketorolac 0,5 mg/kg Q6 hrs, and IV ondansetron $4 \mathrm{mg}$ Q6 hrs. The transition to oral medication (normally on POD3 to 4) was decided by the APS team based on the pain intensity, ambulation status, adverse effects, etc. Postoperative medication data were recorded in the patients' electronic medical charts.

\section{Acute postoperative opioid consumption}

Hourly opioid consumption during the acute postoperative period (POH1-POH120) was extracted from patients' electronic medication administration records. To establish analgesic equivalency, all opioids were converted to oral morphine equivalents (OMEQ) according to Coté et $\mathrm{al}^{14}$ and the Compendium of Pharmaceutical \& Specialties (Canadian Pharmacists Association), ${ }^{15}$ and cumulative and noncumulative 6-hr opioid intakes were calculated.

\section{Acute postoperative pain assessment}

Self-reported pain intensity was assessed using the Numerical Rating Scale (NRS 0-10), where 0 indicated no pain and 10 indicated the worst pain imaginable. The NRS has been validated in the pediatric population and is recommended for use in clinical research. ${ }^{16,17}$ During the acute postoperative period (POH1-POH120), self-reported NRS pain scores were collected periodically by ward nurses. Scores were extracted from patients' electronic medical charts and average postoperative $24 \mathrm{hr}$ day (POD) pain intensities were calculated.

\section{Statistical analysis}

Growth mixture modeling (GMM) was used to conduct acute postoperative opioid consumption trajectory analyses in accordance with other studies. ${ }^{10,18}$ GMM evaluates longitudinal data for interindividual variability in intraindividual patterns of change over time. As populations are not homogenous and subgroups are not always observed, GMM allows for the identification and simultaneous modeling of trajectories of multiple latent subgroups in which variation within trajectory groups is accounted for. ${ }^{19}$ Cumulative 6-hr opioid consumption from POH1 to POH120 were used in the analyses (a total of 20 measurements). Twelve opioid consumption trajectory models were tested using the latent class mixed model (lcmm) package in $\mathrm{R}$ version 3.2.1. Variations between models were influenced by the number of classes (1-6) and shape (linear or quadratic) of the trajectories. Selection of the final model was based on the goodness of fit criteria Akaike information criterion (AIC) and Bayesian information criterion (BIC) (lower values indicating better fit), high entropy of $>0.8$ (reflecting high confidence of group membership), a minimum trajectory class size of $5 \%$ of total study population, parsimony and theoretical soundness. ${ }^{10,19}$ Identified trajectories were characterized by their intercept and slopes.

After final model selection, pre- and intraoperative variables were individually entered in the model as predictors of trajectory membership, including age, sex, pain (in the last 6 months, in the last 1 month and back pain at rest), preoperative pain medication use, length of surgery and intraoperative anesthetic total doses. Significant predictors were determined using the Wald test, where a Wald $P$-value $<0.05$ indicated a variable that was able to explain the difference in slope and/or intercept between 2 trajectories. As such, the Wald test identified variables that were predictive of opioid trajectories with flatter versus steeper slopes or lower versus higher intercepts. Significant variables at the individual level were entered to yield the final model.

Univariate analysis of variance (ANOVA) or KruskalWallis $\mathrm{H}$ test and chi-squared test $(\chi 2)$ were used accordingly with IBM SPSS Statistics to evaluate significant differences between high/low opioid consumers in pain, functional activity and pain medication use at 6 months after surgery. Significant differences in ANOVA or Kruskal-Wallis $\mathrm{H}$ test were followed by appropriate post hoc tests. 
The required sample size for GMM was based on the theoretical foundations of the study, characteristics of the data, measurement reliability and group differences. ${ }^{19,20}$ It is accepted that a minimum sample size of 100 is required for GMM. ${ }^{19,20}$

\section{Results}

\section{Study population}

The final study population consisted of 106 patients (81 females [76\%] and 25 males [24\%]) with a mean age of $15.4 \pm 2.0$ years. Within the last 6 months prior to surgery, 9 patients $(9 \%)$ reported no pain, 34 patients $(32 \%)$ experienced mild pain and 62 patients (59\%) experienced moderate to severe pain and. Within the last 1 month prior to surgery, 29 patients $(27 \%)$ experienced mild pain, 64 patients $(61 \%)$ experienced moderate to severe pain and 13 patients (12\%) reported no pain. Only 17 patients (16\%) reported no back pain at rest. Forty-five patients (42.5\%) were able to perform full activities without restriction and 30 patients $(28 \%)$ reported missing school and/or work days due to back pain in the last 3 months. Thirty-two patients $(30 \%)$ were taking medications for pain, with 6 patients taking opioids. Preoperative and intraoperative characteristics are summarized in Table 1.

\section{Opioid consumption}

Opioid consumption was highest during the first 3 days following surgery, peaking on POD2 with a mean intake of $0.16 \pm 0.06 \mathrm{mg} / \mathrm{kg} / \mathrm{hr}$ (Figure 1A). On average, PCA use was discontinued at the end of POD3, 65 $\pm 9.4 \mathrm{hrs}$ after surgery. On POD4, opioid consumption declined sharply (mean intake of $0.06 \pm 0.04 \mathrm{mg} / \mathrm{kg} / \mathrm{hr}$ ) and remained low until discharge on POD5 (mean intake of $0.05 \pm 0.03 \mathrm{mg}$ / $\mathrm{kg} / \mathrm{hr}$ ). Mean cumulative opioid consumption $120 \mathrm{hrs}$ after surgery was $13.23 \pm 5.20 \mathrm{mg} / \mathrm{kg}$ (Figure 1B).

\section{Pain intensity}

In contrast to opioid consumption, patients experienced consistent pain for the duration of the acute postoperative period. Pain intensity ranged from $3.3 \pm 1.6$ on POD1 to 4.1 \pm 1.7 on POD4 (Figure 1C).

\section{Trajectories}

Twelve models were tested using GMM based on a combination of 1 to 6 trajectory classes and the presence or absence of a quadratic term. Goodness of fit indices for tested models are presented in Table 2. The model with the best fit
Table I Descriptive statistics of preoperative and intraoperative variables of study participants $(n=106)$

\begin{tabular}{|c|c|}
\hline Patient characteristics & $\begin{array}{l}\text { Total patient } \\
\text { sample }\end{array}$ \\
\hline \multicolumn{2}{|l|}{ Demographics } \\
\hline Age, years & $15.4(2.0)$ \\
\hline \multicolumn{2}{|l|}{ Sex, n (\%) } \\
\hline Female & $81(76)$ \\
\hline Male & $25(24)$ \\
\hline \multicolumn{2}{|l|}{ Preoperative SRS-30 variables, n (\%) } \\
\hline \multicolumn{2}{|l|}{ Pain in last 6 months } \\
\hline None & $9(9)$ \\
\hline Mild & $34(32)$ \\
\hline Moderate to severe & $62(59)$ \\
\hline \multicolumn{2}{|l|}{ Pain in last I month } \\
\hline None & $13(12)$ \\
\hline Mild & $29(27)$ \\
\hline Moderate to severe & $64(6 \mathrm{I})$ \\
\hline \multicolumn{2}{|l|}{ Back pain at rest } \\
\hline No & $17(16)$ \\
\hline Yes & $89(84)$ \\
\hline \multicolumn{2}{|l|}{ Current level of activity } \\
\hline Full activities without restriction & $45(42.5)$ \\
\hline Light to moderate activity & $45(42.5)$ \\
\hline No activity & $16(15)$ \\
\hline \multicolumn{2}{|l|}{$\begin{array}{l}\text { Missed school/work days due to back } \\
\text { pain in last } 3 \text { months }\end{array}$} \\
\hline 0 & $75(72)$ \\
\hline $\mathrm{I}-3$ & $17(16)$ \\
\hline 4 or more & $13(12)$ \\
\hline \multicolumn{2}{|l|}{ Medications for pain, $\mathrm{n}(\%)$} \\
\hline None & $74(70)$ \\
\hline \multicolumn{2}{|l|}{ Yes } \\
\hline Non-opioids & $26(24)$ \\
\hline Opioids & $6(6)$ \\
\hline \multicolumn{2}{|l|}{ Anesthetic/Analgesia during surgery } \\
\hline Propofol mg/kg & $52.5(12.4)$ \\
\hline Remifentanil, $\mu g / \mathrm{kg}$ & $45.6(22.9)$ \\
\hline Sufentanil, $\mu g / \mathrm{kg}$ & $0.15(0.36)$ \\
\hline Fentanyl, $\mu g / k g$ & $0.30(0.79)$ \\
\hline Dexamethasone $\mathrm{mg} / \mathrm{kg}$ & $0.11(0.04)$ \\
\hline Ketamine mg/kg & $0.70(0.57)$ \\
\hline Intrathecal morphine, $\mu \mathrm{g} / \mathrm{kg}$ & $5.40(2.97)$ \\
\hline Total intraoperative opioids, mg/kg & $36.1(17.0)$ \\
\hline \multicolumn{2}{|l|}{ Surgical variables } \\
\hline Surgery length, minutes & $264(76.4)$ \\
\hline Blood loss, $\mathrm{mL}$ & $76 I(44 I)$ \\
\hline Number of fused vertebrae & $10.7(2.50)$ \\
\hline
\end{tabular}

Notes: Data are presented as mean $\pm S D$ in parenthesis, unless otherwise stated. Abbreviation: SRS-30, Scoliosis Research Society Questionnaire: Version 30.

$(\mathrm{AIC}=6708.04, \mathrm{BIC}=6761.31)$ contained 5 cumulative opioid consumption trajectories, a quadratic term, entropies of $>0.8$ for all patients and a smallest class size of $7.5 \%$ of the total study 
A

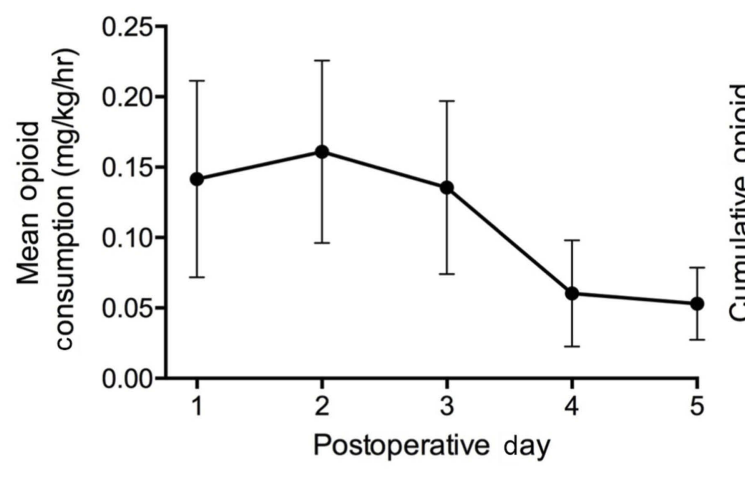

C
B

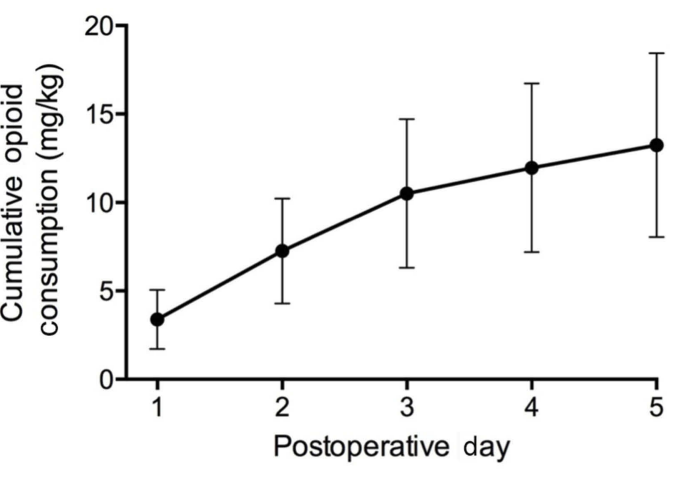

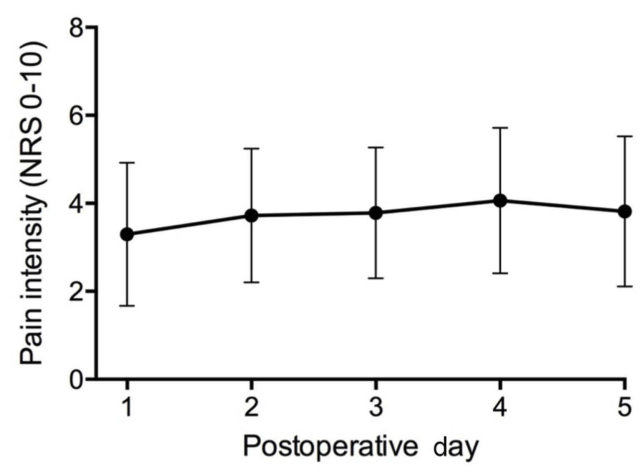

Figure I Postoperative mean opioid consumption (A), cumulative opioid consumption $(\mathbf{B})$ and pain intensity $(\mathbf{C})$ of study participants $(n=106)$. Data are presented as mean \pm SD. Abbreviation: NRS, numeric rating scale.

population. Preoperative and intraoperative factors were entered into the model and tested as predictors of opioid trajectory membership. Intraoperative intrathecal morphine dose was found to be the only significant predictor of trajectory membership, explaining the variation between opioid trajectories 4 and 5 (Wald $\chi^{2} \mathrm{p}=0.0498$ ). As such, the final model (AIC $=6703.26$, $\mathrm{BIC}=6767.19)$ included intrathecal morphine dose as a predictor and the structure and characteristics of the regression equation for each trajectory are presented in Figure 2 and Table 3, respectively.

For all opioid trajectories, rate of opioid consumption was highest in the initial days following surgery. In ascending order, opioid trajectories 1 to 5 differed in the total amount of opioids consumed (from low to high consumption) and the rate of opioid intake (from low to high rate of intake) during the acute postoperative period. As such, opioid trajectory $1(\mathrm{n}=19)$ was characterized by patients with the lowest rate of opioid consumption, which resulted in the lowest cumulative opioid intake by POD5. Opioid trajectory $5(\mathrm{n}=8)$ was characterized by patients with the highest rate of opioid consumption, which resulted in the highest cumulative opioid intake by POD5. Overall, 2 populations of patients were identified: high opioid consumers (trajectories 4 and 5) and low opioid consumers (trajectories 1,2 and 3). No significant difference was found between high/low opioid consumers and preoperative and intraoperative factors (Table 4). Acute postoperative pain according to opioid trajectory membership is presented in Figure 3.

\section{Long-term outcomes}

At the 6-month follow-up appointment, 15 patients (20\%) reported no pain within the last 6 months and 24 patients $(32 \%)$ reported no pain within the last 1 month. Of the patients that reported pain within the last 6 months, 30 patients (39\%) experienced mild pain while 31 patients (41\%) experienced moderate to severe pain. Within the last 1 month, 32 patients (42\%) experienced mild pain while only 20 patients (26\%) experienced moderate to severe pain. Moreover, 26 patients (34\%) had no back pain at rest. Seven patients (9\%) stated that they were able to engage in full activities without restriction. However, 21 patients (28\%) missed school and/or work due to back pain in the last 3 months. Lastly, patients stated reduced medication use for pain, with 4 patients $(5 \%)$ taking opioids and 22 patients (29\%) taking non-opioid medications. 
Table 2 Trajectory model goodness of fit indices

\begin{tabular}{|c|c|c|c|c|c|c|}
\hline \multirow[t]{3}{*}{ Number of trajectories } & \multicolumn{6}{|c|}{ Opioid trajectory } \\
\hline & \multicolumn{3}{|l|}{ Linear } & \multicolumn{3}{|c|}{ Linear + quadratic } \\
\hline & AIC & BIC & SC (\%) & AIC & BIC & SC (\%) \\
\hline I & $11,661.17$ & $11,669.16$ & 100 & $\mathrm{II}, 558.96$ & $11,569.62$ & 100 \\
\hline 2 & 9815.50 & 9831.48 & 29.2 & 9512.10 & 9533.41 & 29.2 \\
\hline 3 & 8865.47 & 8889.44 & 22.6 & 8321.96 & 8353.92 & 22.6 \\
\hline 4 & 8378.71 & 8410.67 & 17.9 & 7642.71 & 7685.33 & 17.9 \\
\hline 5 & 7793.72 & 7833.67 & 7.5 & $6708.04 *$ & $6761.31 *$ & 7.5* \\
\hline 6 & 7560.05 & 7607.99 & 2.8 & 6274.58 & 6338.51 & 2.8 \\
\hline
\end{tabular}

Notes: *Indicates model with the best fit based on lowest Akaike information criterion (AIC) and Bayesian information criterion (BIC), $>0.80$ entropy (reflecting high confidence of group membership), a minimum trajectory class size of $5 \%$ of total study population, parsimony and theoretical soundness.

Abbreviation: AIC, Akaike information criterion; BIC, Bayesian information criterion; SC, smallest class size.

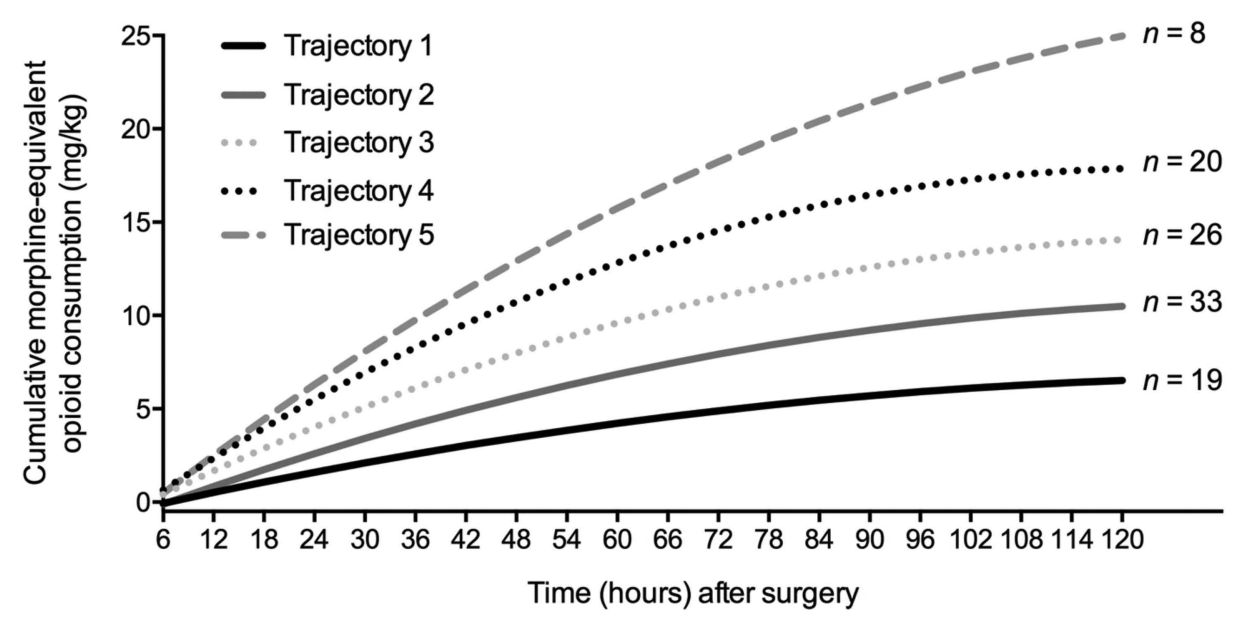

Figure 2 Acute postoperative opioid consumption trajectories. The final model included intraoperative intrathecal morphine as a predictor of opioid trajectory membership $(\mathrm{AIC}=6703.26$ and $\mathrm{BIC}=6767.19)$.

Table 3 Description of final opioid trajectory model and predicted cumulative opioid consumption

\begin{tabular}{|l|l|l|l|l|l|l|l|l|l|}
\hline & & \multicolumn{3}{|l|}{ Slopes } & \multicolumn{3}{l|}{ Predicted values (mg/kg) } \\
\hline $\begin{array}{l}\text { Opioid } \\
\text { trajectory }\end{array}$ & $\mathbf{n}$ & Intercept & Linear & Quadratic & $\begin{array}{l}\text { PODI } \\
\mathbf{( 2 4} \text { hrs) }\end{array}$ & $\begin{array}{l}\text { POD2 } \\
\text { (48 hrs) }\end{array}$ & $\begin{array}{l}\text { POD3 } \\
\text { (72 hrs) }\end{array}$ & $\begin{array}{l}\text { POD4 } \\
\text { (96 hrs) }\end{array}$ & $\begin{array}{l}\text { POD5 } \\
\text { (I 20 hrs) }\end{array}$ \\
\hline 1 & 19 & -0.66287 & 0.10306 & -0.00036 & 1.60 & 3.45 & 4.89 & 5.91 & 6.52 \\
2 & 33 & -1.10752 & 0.16866 & -0.00060 & 2.59 & 5.61 & 7.93 & 9.55 & 10.49 \\
3 & 26 & -0.90853 & 0.22558 & -0.00084 & 4.02 & 7.98 & 10.98 & 13.01 & 14.06 \\
4 & 20 & -1.15803 & 0.30732 & -0.00124 & 5.50 & 10.74 & 14.54 & 16.92 & 17.86 \\
5 & 8 & -1.62623 & 0.35726 & -0.00113 & 6.30 & 12.92 & 18.24 & 22.26 & 24.97 \\
\hline
\end{tabular}

Abbreviations: POD, postoperative day; hrs, hours.

\section{Opioid consumption trajectory membership and long-term outcomes}

No significant differences were found between high opioid consumers (trajectories 4 and 5) and low opioid consumers (trajectories 1,2 and 3) in pain (in the last 6 months, in the last 1 month and back pain at rest), functional activity (current level of activity and missed school/work days) or pain medication use at 6 months after surgery (Table 5). 
Table 4 Preoperative and intraoperative factors according to high/low opioid consumer membership

\begin{tabular}{|c|c|c|c|c|c|}
\hline \multirow[b]{2}{*}{ Preoperative and intraoperative factors } & \multicolumn{3}{|c|}{ Low opioid consumer } & \multicolumn{2}{|c|}{ High opioid consumer } \\
\hline & $\begin{array}{l}\text { Traj I } \\
(n=19)\end{array}$ & $\begin{array}{l}\text { Traj } 2 \\
(n=33)\end{array}$ & $\begin{array}{l}\text { Traj } 3 \\
(n=26)\end{array}$ & $\begin{array}{l}\text { Traj } 4 \\
(n=20)\end{array}$ & $\begin{array}{l}\text { Traj } 5 \\
(n=8)\end{array}$ \\
\hline \multicolumn{6}{|l|}{ Demographics } \\
\hline Age, years & $15.4(2.0)$ & $15.0(1.9)$ & $15.9(2.1)$ & I5.7 (I.8) & I5.I (2.2) \\
\hline \multicolumn{6}{|l|}{ Sex, $n(\%)$} \\
\hline Female & $13(68)$ & $27(82)$ & $17(65)$ & $18(90)$ & $6(75)$ \\
\hline Male & $6(32)$ & $6(18)$ & $9(35)$ & $2(10)$ & $2(25)$ \\
\hline \multicolumn{6}{|l|}{ Preoperative SRS-30 variables, $n$ (\%) } \\
\hline \multicolumn{6}{|l|}{ Pain in last 6 months } \\
\hline None & $2(10)$ & $3(9)$ & $3(12)$ & $0(0)$ & I (I2.5) \\
\hline Mild & $7(37)$ & II (33) & $7(28)$ & $8(40)$ & I (I2.5) \\
\hline Moderate to severe & $10(53)$ & $19(58)$ & $15(60)$ & $12(60)$ & $6(75)$ \\
\hline \multicolumn{6}{|l|}{ Pain in last I month } \\
\hline None & $3(16)$ & $3(9)$ & $5(19)$ & I (5) & I (I2.5) \\
\hline Mild & $6(31)$ & $10(30)$ & $7(27)$ & $6(30)$ & $0(0)$ \\
\hline Moderate to severe & $10(53)$ & $20(6 \mathrm{I})$ & $14(54)$ & $13(65)$ & 7 (87.5) \\
\hline \multicolumn{6}{|l|}{ Back pain at rest } \\
\hline No & $4(2 I)$ & $7(2 I)$ & $3(12)$ & $2(10)$ & I (I2.5) \\
\hline Yes & $15(79)$ & $26(79)$ & $23(88)$ & $18(90)$ & $7(87.5)$ \\
\hline \multicolumn{6}{|l|}{ Current level of activity } \\
\hline Full activities without restriction & $10(53)$ & $16(49)$ & $8(3 I)$ & $8(40)$ & $3(37.5)$ \\
\hline Light to moderate activity & $8(42)$ & II (33) & $15(58)$ & $7(35)$ & $4(50)$ \\
\hline No activity & I (5) & $6(18)$ & $3(I I)$ & $5(25)$ & I (I2.5) \\
\hline \multicolumn{6}{|c|}{ Missed school/work days due to back pain in last 3 months } \\
\hline 0 & $14(74)$ & $25(76)$ & $18(69)$ & $12(60)$ & $6(86)$ \\
\hline $1-3$ & $3(16)$ & $4(12)$ & $6(23)$ & $3(15)$ & I (I4) \\
\hline 4 or more & $2(10)$ & $4(12)$ & $2(8)$ & $5(25)$ & $0(0)$ \\
\hline \multicolumn{6}{|l|}{ Medications for pain, $n$ (\%) } \\
\hline None & $12(63)$ & $26(79)$ & $19(73)$ & $12(60)$ & $5(63)$ \\
\hline \multicolumn{6}{|l|}{ Yes } \\
\hline Non-opioids & $5(26)$ & $6(18)$ & $6(23)$ & $7(35)$ & $2(25)$ \\
\hline Opioids & $2(\mathrm{II})$ & I (3) & I (4) & I (5) & I (I3) \\
\hline \multicolumn{6}{|l|}{ Intraoperative factors } \\
\hline Surgery length, mins & $249(67.8)$ & $26 \mid(63.5)$ & $285(91.1)$ & $256(73.0)$ & $274(103)$ \\
\hline Propofol, mg/kg & $47.3(12.6)$ & $51.8(12.1)$ & $54.3(11.6)$ & $53.5(14.3)$ & $58.7(9.04)$ \\
\hline Remifentanil, $\mu \mathrm{g} / \mathrm{kg}$ & $42.2(22.8)$ & $43.0(21.6)$ & $50.5(25.8)$ & $44.3(22.2)$ & $50.4(21.6)$ \\
\hline Sufentanil, $\mu g / k g$ & $0.19(0.45)$ & $0.12(0.26)$ & $0.05(0.12)$ & $0.32(0.58)$ & $0.03(0.08)$ \\
\hline Fentanyl, $\mu g / k g$ & $0.24(0.75)$ & $0.16(0.68)$ & $0.42(0.90)$ & $0.33(0.80)$ & $0.54(0.99)$ \\
\hline Intrathecal morphine, $\mu g / \mathrm{kg}$ & $4.77(1.03)$ & $6.65(4.36)$ & $4.86(1.70)$ & $4.37(1.35)$ & $6.10(3.73)$ \\
\hline Total surgical opioids, mg/kg & $33.5(17.1)$ & $34.6(16.5)$ & $39.6(19.1)$ & $34.9(16.3)$ & $39.8(15.2)$ \\
\hline
\end{tabular}

Notes: Data are presented as mean $\pm S D$ in parenthesis, unless otherwise stated.

Abbreviation: Traj, trajectory; SRS-30, Scoliosis Research Society Questionnaire: Version 30.

\section{Discussion}

The objectives of this study were to investigate acute postoperative opioid consumption trajectories, their predictors and associations with long-term outcomes in adolescents undergoing posterior spinal fusion surgery. Five opioid consumption trajectories were identified during the in-hospital period, reflecting latent subgroups within this pediatric population. Specifically, two types of patients' behavior were identified: high opioid consumers (trajectories 4 and 5), and low opioid consumers (trajectories 1, 2 




Figure 3 Acute postoperative pain intensity according to opioid consumption trajectory membership (B). Pain intensities are presented as mean \pm SD. ANOVA followed by Tukey-Kramer tests were performed for all postoperative days. A significant difference $(p<0.00 \mathrm{I})$ between ${ }^{*}$ trajectories I and 3 , ${ }^{\ddagger}$ trajectories I and 4 , ${ }^{\dagger}$ trajectories 2 and 4 ,

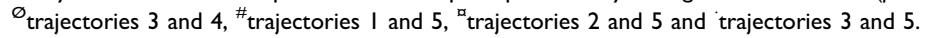

Abbreviation: NRS, numeric rating scale.

and 3). The dose of intrathecal morphine received during surgery was a predictor of opioid trajectory membership. Importantly, results revealed that high/low opioid consumption behavior was not associated with pain, functional activity or pain medication use (opioids and nonopioids) 6 months after surgery.

The results of our study revealed that opioid consumption rate varied in the acute postoperative period, with higher opioid intake in the immediate days following surgery. In a similar population of adolescents with idiopathic scoliosis, Kwan et $\mathrm{al}^{9}$ found that the use of PCA morphine decreased at $12 \mathrm{hr}$ intervals following spinal fusion surgery. Together, our results suggest that opioid consumption in the acute postoperative period is indeed dynamic and evolves with time. A more precise representation of a patient's opioid consumption may offer better insight into progression to long-term outcomes and may have more meaningful implications on patients care than traditional mean measures. ${ }^{8}$

It is important to note that in the study conducted by Kwan et al, ${ }^{9}$ opioid intake at each timepoint showed substantial variability, pointing to a heterogenous study population. In fact, this finding is consistent across numerous studies that examined opioid consumption in the days after surgery, despite stringent inclusion and exclusion criteria of study participants such as age and BMI restrictions, exclusion of chronic illnesses and exclusion of patients that deviated from intraoperative anesthesia protocol. ${ }^{10,21}$ Through the use of GMM, we demonstrated for the first time that 5 latent subgroups exist in the pediatric population with regards to opioid intake. These subgroups differed in their rate of opioid consumption and total amount of opioids consumed. The presence of multiple opioid trajectories reveals interindividual differences in opioid intake after surgery due to unanticipated factors. This may explain the variability found by previous research groups. From a clinical perspective, knowledge of interindividual differences allows for better anticipation of inhospital opioid-related adverse events.

Something to consider is the amount of pain experienced by patients after surgery. We found that on average, high opioid consumers reported significantly greater pain than low opioid consumers. Specifically, patients in opioid trajectory 1 demonstrated mild pain $(\mathrm{NRS}<3$ ) throughout the 5 postoperative days. In accordance, these patients reported the lowest rate and amount of opioids consumed after surgery. Conversely, patients in trajectory 5 reported moderate pain (NRS $>4$ ) immediately after surgery, that increased over the duration of their in-hospital stay, peaking at $5.9 \pm 1.1$. These patients reported the highest rate and amount of opioids consumed during this time. Such positive association between opioid consumption and pain has

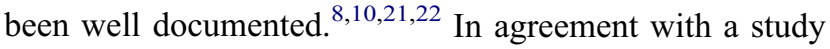
including 1,736 adults, patients who experienced NRS $>4$ of pain intensity after awakening from surgery consumed significantly more opioids in the PACU than patients who reported lower pain. ${ }^{21}$ It is therefore possible that immediate postsurgical pain may in part explain the presence of multiple opioid trajectories. Clinically, patients who are reporting greater pain after surgery may need to be monitored more closely for opioid-related adverse events in hospital than patients reporting less pain. Indeed, the amount of pain experienced immediately after surgery may be influenced by the extent of surgical intervention performed. However, in a similar cohort of AIS patients undergoing posterior spinal fusion surgery, Ferland et $\mathrm{al}^{23}$ 
Table 5 Long-term outcomes after surgery according to high/low opioid consumer membership

\begin{tabular}{|c|c|c|c|c|c|c|}
\hline \multirow[b]{2}{*}{ Long-term outcomes } & \multicolumn{3}{|c|}{$\begin{array}{l}\text { Low opioid } \\
\text { consumer }\end{array}$} & \multicolumn{2}{|c|}{$\begin{array}{l}\text { High opioid } \\
\text { consumer }\end{array}$} & \multirow[t]{2}{*}{$\begin{array}{l}\chi_{(\mathrm{d})}^{2} \\
\text { (P-value) }\end{array}$} \\
\hline & $\begin{array}{l}\text { Traj I } \\
(n=16)\end{array}$ & $\begin{array}{l}\text { Traj } 2 \\
(n=18)\end{array}$ & $\begin{array}{l}\text { Traj } 3 \\
(n=22)\end{array}$ & $\begin{array}{l}\text { Traj } 4 \\
(n=\mid 4)\end{array}$ & $\begin{array}{l}\text { Traj } 5 \\
(n=6)\end{array}$ & \\
\hline \multicolumn{7}{|l|}{6 Month SRS-30 variables, $n(\%)$} \\
\hline Pain in last 6 months & & & & & & $2.785_{(2)}(0.248)$ \\
\hline None & $6(37.5)$ & $4(22)$ & $3(14)$ & I (7) & $\mathrm{I}(\mathrm{I7})$ & \\
\hline Mild & $4(25)$ & 7 (39) & $12(54)$ & $4(29)$ & $3(50)$ & \\
\hline Moderate to severe & $6(37.5)$ & 7 (39) & $7(32)$ & $9(64)$ & $2(33)$ & \\
\hline Pain in last I month & & & & & & $5.852_{(2)}(0.054)$ \\
\hline None & $7(44)$ & 7 (39) & $8(36.4)$ & I (7) & I (I7) & \\
\hline Mild & $7(44)$ & $6(33)$ & $8(36.4)$ & $8(57)$ & $3(50)$ & \\
\hline Moderate to severe & $2(12)$ & $5(28)$ & $6(27.2)$ & $5(36)$ & $2(33)$ & \\
\hline Back pain at rest & & & & & & $2.435_{(1)}(0.119)$ \\
\hline No & $6(37.5)$ & $6(33)$ & $10(45)$ & $3(2 I)$ & I (I7) & \\
\hline Yes & $10(62.5)$ & $12(67)$ & $12(55)$ & II (79) & $5(83)$ & \\
\hline Current level of activity & & & & & & $1.150_{(2)}(0.563)$ \\
\hline Full activities without restriction & $2(12.5)$ & $2(11)$ & $0(0)$ & $0(0)$ & $3(50)$ & \\
\hline Light to moderate activity & $12(75)$ & $14(78)$ & $21(95)$ & $12(86)$ & $3(50)$ & \\
\hline No activity & $2(12.5)$ & $2(I I)$ & I (5) & $2(14)$ & $0(0)$ & \\
\hline Missed school/work days due to back pain in last 3 months & & & & & & $3.930_{(2)}(0.140)$ \\
\hline 0 & $13(87)$ & I5 (83.3) & $15(68)$ & $9(64)$ & $2(33.3)$ & \\
\hline $1-3$ & $2(13)$ & $2(11.1)$ & $3(14)$ & $3(22)$ & $2(33.3)$ & \\
\hline 4 or more & $0(0)$ & I (5.6) & $4(18)$ & $2(14)$ & $2(33.3)$ & \\
\hline Medications for pain, $n(\%)$ & & & & & & $3.007_{(1)}(0.083)$ \\
\hline None & $10(62.5)$ & $12(66.6)$ & $18(82)$ & $8(57)$ & $2(33)$ & \\
\hline Yes & & & & & & \\
\hline Non-opioids & $6(37.5)$ & $5(27.8)$ & $3(14)$ & $5(36)$ & $3(50)$ & \\
\hline Opioids & $0(0)$ & I (5.6) & I (4) & I (7) & I (I7) & \\
\hline
\end{tabular}

Notes: Data are presented as $n$ (\%). Between high/low opioid consumers, Pearson's chi-squared test was performed for all variables. Abbreviation: Traj, trajectory; SRS-30, Scoliosis Research Society Questionnaire: Version 30.

found that the number of vertebrae fused during surgery was not significantly associated with acute postoperative pain. Interestingly, our findings showed that despite high opioid consumption, adolescents in opioid trajectory 5 experienced moderate pain that continued to increase up until POD5. In these patients, the analgesic effect of opioids may not has been sufficient enough to provide adequate pain control. This affirms the need for more personalized modes of pain management.

In our study, the dose of intrathecal morphine received during surgery was a predictor of opioid trajectory membership, delineating high opioid consumers into two groups. A low dose of intrathecal morphine was associated with membership in trajectory 4, while a high dose was associated with membership in trajectory 5. In adult patients who underwent spinal fusion surgery, Urban et $\mathrm{al}^{24}$ demonstrated that low intrathecal morphine doses led to significantly more postsurgical pain and PCA morphine use within the first 12 hrs after surgery than high intrathecal morphine doses. While not statistically significant, across all trajectories, patients in opioid trajectory 4 received the lowest dose of intrathecal morphine and reported the highest level of pain on POD1. Understandably, these patients also reported significantly higher rates of opioid consumption than low opioid consumers. Therefore, opioid trajectory 4 may be a result of low intraoperative intrathecal morphine that was unable to protect against acute postoperative pain and subsequent opioid use. Contradicting the findings of Urban et al, ${ }^{24}$ patients in trajectory 5 received a high dose of intrathecal morphine, but reported the highest rate of opioid intake and total opioid consumed in the 5 days after surgery. It can be hypothesized that this small subset of patients $(n=8)$ did not respond to the analgesic effects of opioids (both intraoperative intrathecal morphine or postoperative 
opioids), perhaps due to pharmacogenetic variations, resulting in high opioid intake and minimal benefit on pain relief. For instance, it has been shown that individuals with a G/G genotype for the opioid receptor $\mu 1$ rs1799971 single nucleotide polymorphism reported greater PCA morphine use after surgery than A/A individuals. ${ }^{25}$ Fortunately, of the total study population, less than $8 \%$ of patients (trajectory 5) appear to be opioid non-responders.

We found that high/low opioid consumption behavior was not significantly associated with persistent postsurgical pain at 6 months after surgery. Therefore, acute postoperative opioid consumption may not predict the development of persistent postsurgical pain. In contrast to our findings, Julien-Marsollier et $\mathrm{al}^{26}$ found a positive association between morphine consumption on POD1 and 3 and chronic neuropathic pain in a similar cohort of AIS patients. The discrepancy in findings may be due to the dynamic versus static measures of acute postoperative opioid consumption. Furthermore, while the latter study focused on chronic neuropathic pain, we did not differentiate between nociceptive or neuropathic pain.

Importantly, use of opioid and non-opioid pain medications was not associated with high/low opioid consumers at 6 months after surgery. Moreover, the proportion of patients using opioids before and 6 months after surgery remained constant. This finding broadly disagrees with current literature. In their retrospective cohort study of pediatric patients after abdominal surgery, Owusu-Agyemang et $\mathrm{al}^{8}$ showed that average postoperative opioid consumption was significantly associated with persistent opioid use at 6 months after surgery. This lack of consensus may be explained by the fact that existing literature examines acute postoperative opioid consumption and its association with long-term outcomes using single timepoint or mean measures of opioid consumption as opposed to a continuous measure. Our findings suggest that in children and adolescents, opioid consumption during the acute postoperative period is not associated with long-term opioid use. This notion challenges the current belief that greater opioid consumption after surgery leads to greater risk of opioid misuse.

While it is known that long-term pain leads to delayed rehabilitation and physical recovery, ${ }^{27}$ our results showed that opioid trajectory membership was not associated with functional activity 6 months after surgery. Thus, analgesic consumption in the acute postoperative period does not influence functional outcome after surgery. Altogether, the lack of association between acute postoperative opioid consumption and long-term patient outcomes suggest that clinically, while in-hospital opioid use should be closely controlled for adverse events, less caution in limiting acute opioid exposure may be warranted for preventing longterm patient consequences.

There were several limitations in our study. First, while there is a well-established link between opioid consumption and pain, acute postoperative pain was not tested as a predictor of opioid trajectory membership. Second, anticipation of analgesic benefit was not considered as a predictor of opioid consumption after surgery. Positive expectation of opioid efficacy has been shown to enhance the analgesic benefit via changes in endogenous pain modulation. ${ }^{28}$ As such, while a multitude of variables were investigated as factors that may influence opioid consumption, the study nevertheless was unable to account for all possible variables. Third, following PCA discontinuation, opioid doses were standardized $(\mathrm{mg} / \mathrm{kg})$, allowing individualization only through rescue doses for breakthrough pain. Finally, while the concentration of intrathecal morphine was suggested to $5 \mu \mathrm{g} / \mathrm{kg}$ in our study, differences were observed, while not statistically significant, in the concentration of intrathecal morphine received by patients in different opioid trajectories. Consequently, our findings suggest that the clinical judgment of anesthesiologists may have a lasting impact on patients' acute postoperative experience. Future studies can be conducted to evaluate postoperative pain as a time-variant predictor of opioid trajectories and anticipation of analgesic benefit as a time-invariant predictor of opioid trajectories.

\section{Conclusion}

In this study of adolescents undergoing posterior spinal fusion surgery, 5 acute postoperative opioid consumption trajectories were identified that could be broadly divided into high opioid consumers and low opioid consumers. Intraoperative intrathecal morphine dose was a significant predictor of opioid trajectory membership. Notably, high/low opioid consumers were not associated with pain, functional activity or use of pain medications (opioids and non-opioids) 6 months after surgery. Future studies examining the role of pharmacogenetic variations and pain on opioid consumption trajectories are required.

\section{Author contributions}

All authors contributed to data analysis, drafting or revising the article, gave final approval of the version to be published, and agree to be accountable for all aspects of the work. 


\section{Ethics statement}

This study received ethics approval prior to the beginning of the study from the Research Ethics Board of McGill University (A08-M71-17B).

\section{Acknowledgments}

The study received financial support from the Shriners Hospitals for Children (71004-CAN-17), the Montreal Children's Hospital Foundation and the McGill Scoliosis \& Spinal Research Chair.

\section{Disclosure}

Dr. Jean A. Ouellet reports grants from the AO foundation, outside the submitted work. The authors report no other conflicts of interest in this work.

\section{References}

1. Schoenfeld AJ, Nwosu K, Jiang W, et al. Risk factors for prolonged opioid use following spine surgery, and the association with surgical intensity, among opioid-naive patients. J Bone Joint Surg Am. 2017;99(15):1247-1252. doi:10.2106/JBJS.16.01075

2. Cauley CE, Anderson G, Haynes AB, Menendez M, Bateman BT, Ladha K. Predictors of in-hospital postoperative opioid overdose after major elective operations: a nationally representative cohort study. Ann Surg. 2017;265(4):702-708. doi:10.1097/SLA.0000000000001945

3. Calcaterra SL, Scarbro S, Hull ML, Forber AD, Binswanger IA, Colborn KL. Prediction of future chronic opioid use among hospitalized patients. J Gen Intern Med. 2018;33(6):898-905. doi:10.1007/ s11606-018-4335-8

4. Wright JG, Menaker RJ, Canadian Paediatric Surgical Wait Times Study G. Waiting for children's surgery in Canada: the Canadian Paediatric Surgical Wait Times project. Cmaj. 2011;183(9):E559E564. doi:10.1503/cmaj.101530

5. Tzong KY, Han S, Roh A, Ing C. Epidemiology of pediatric surgical admissions in US children: data from the HCUP kids inpatient database. J Neurosurg Anesthesiol. 2012;24(4):391-395. doi:10. 1097/ANA.0b013e31826a0345

6. Connelly M, Fulmer RD, Prohaska J, et al. Predictors of postoperative pain trajectories in adolescent idiopathic scoliosis. Spine (Phila Pa 1976). 2014;39(3):E174-E181. doi:10.1097/BRS.0000000000000099

7. Rabbitts JA, Zhou C, Groenewald CB, Durkin L, Palermo TM. Trajectories of postsurgical pain in children: risk factors and impact of late pain recovery on long-term health outcomes after major surgery. Pain. 2015;156(11):2383-2389. doi:10.1097/j.pain.0000000000000281

8. Owusu-Agyemang P, Cata JP, Meter AV, et al. Perioperative factors associated with persistent opioid use after extensive abdominal surgery in children and adolescents: a retrospective cohort study. Paediatr Anaesth. 2018. 28(7):625-631.

9. Kwan MK, Chiu CK, Chan TS, et al. Trajectory of postoperative wound pain within the first 2 weeks following posterior spinal fusion surgery in adolescent idiopathic scoliosis patients. Spine (Phila Pa 1976). 2017;42(11):838-843. doi:10.1097/BRS.0000000000001902

10. Page MG, Katz J, Curtis K, Lutzky-Cohen N, Escobar EM, Clarke HA. Acute pain trajectories and the persistence of post-surgical pain: a longitudinal study after total hip arthroplasty. J Anesth. 2016;30 (4):568-577. doi:10.1007/s00540-016-2183-4
11. Page MG, Karanicolas PJ, Cleary S, et al. In-hospital opioid consumption, but not pain intensity scores, predict 6-month levels of pain catastrophizing following hepatic resection: a trajectory analysis. Eur J Pain. 2019;23 (3):503-514.

12. Parent EC, Hill D, Mahood J, Moreau M, Raso J, Lou E. Discriminative and predictive validity of the scoliosis research society-22 questionnaire in management and curve-severity subgroups of adolescents with idiopathic scoliosis. Spine. 2009;34 (22):2450-2457. doi:10.1097/BRS.0b013e3181af28bf

13. Ghandehari H, Mahabadi MA, Mahdavi SM, Shahsavaripour A, Seyed Tari HV, Safdari F. Evaluation of patient outcome and satisfaction after surgical treatment of adolescent idiopathic scoliosis using scoliosis research society-30. Arch Bone Jt Surg. 2015;3(2):109-113.

14. Charles J. Coté JL RMW, Ralph A. Lugo, Nishan Goudsouzian. In: Coté CJ, ed. A Practice of Anesthesia for Infants and Children.6 ed. Philadelphia, PA: Elsevier; 2019:89-146.

15. AssociAssociation CP. Compendium of Pharmaceuticals \& Specialties: Opioids [product monograph]. https://www.e-therapeutics.ca/. Published October 2017. Accessed July, 1, 2018.

16. Miro J, Castarlenas E, Huguet A. Evidence for the use of a numerical rating scale to assess the intensity of pediatric pain. Eur J Pain. 2009;13(10):1089-1095. doi:10.1016/j.ejpain.2009.07.002

17. Ruskin D, Lalloo C, Amaria K, et al. Assessing pain intensity in children with chronic pain: convergent and discriminant validity of the 0 to 10 numerical rating scale in clinical practice. Pain Res Manag. 2014;19(3):141-148.

18. Page MG, Katz J, Romero Escobar EM, et al. Distinguishing problematic from nonproblematic postsurgical pain: a pain trajectory analysis after total knee arthroplasty. Pain. 2015;156(3):460-468. doi:10.1097/01.j.pain.0000460327.10515.2d

19. Ram N, Grimm KJ. Growth mixture modeling: a method for identifying differences in longitudinal change among unobserved groups. Int $J$ Behav Dev. 2009;33(6):565-576. doi:10.1177/0165025409343765

20. Curran PJ, Obeidat K, Losardo D. Twelve frequently asked questions about growth curve modeling. J Cogn Dev. 2010;11(2):121-136. doi:10.1080/15248371003699969

21. Mei W, Seeling M, Franck M, et al. Independent risk factors for postoperative pain in need of intervention early after awakening from general anaesthesia. Eur J Pain. 2010;14(2):149e141-147. doi:10.1016/j.ejpain.2009.03.009

22. Bot AG, Bekkers S, Arnstein PM, Smith RM, Ring D. Opioid use after fracture surgery correlates with pain intensity and satisfaction with pain relief. Clin Orthop Relat Res. 2014;472(8):2542-2549. doi:10.1007/s11999-014-3660-4

23. Ferland CE, Saran N, Valois T, et al. Preoperative distress factors predicting postoperative pain in adolescents undergoing surgery: a preliminary Study. J Pediatr Health Care. 2017;31(1):5-15. doi:10.1016/j.pedhc.2015.12.008

24. Urban MK, Jules-Elysee K, Urquhart B, Cammisa FP, Boachie-Adjei O. Reduction in postoperative pain after spinal fusion with instrumentation using intrathecal morphine. Spine (Phila Pa 1976). 2002;27(5):535-537.

25. De Gregori M, Diatchenko L, Ingelmo PM, et al. Human genetic variability contributes to postoperative morphine consumption. $J$ Pain. 2016;17(5):628-636. doi:10.1016/j.jpain.2016.02.003

26. Julien-Marsollier F, David R, Hilly J, Brasher C, Michelet D, Dahmani S. Predictors of chronic neuropathic pain after scoliosis surgery in children. Scand J Pain. 2017;17:339-344. doi:10.1016/j.sjpain.2017.09.002

27. Joshi GP, Ogunnaike BO. Consequences of inadequate postoperative pain relief and chronic persistent postoperative pain. Anesthesiol Clin North America. 2005;23(1):21-36. doi:10.1016/j.atc.2004.11.013

28. Bingel U, Wanigasekera V, Wiech K, et al. The effect of treatment expectation on drug efficacy: imaging the analgesic benefit of the opioid remifentanil. Sci Transl Med. 2011;3(70):70ra14. doi:10.1126/ scitranslmed.3001244 


\section{Publish your work in this journal}

The Journal of Pain Research is an international, peer reviewed, open access, online journal that welcomes laboratory and clinical findings in the fields of pain research and the prevention and management of pain. Original research, reviews, symposium reports, hypothesis formation and commentaries are all considered for publication. The manuscript management system is completely online and includes a very quick and fair peer-review system, which is all easy to use. Visit http:// www.dovepress.com/testimonials.php to read real quotes from published authors. 